\title{
Challenges and Progresses of Molecular Plant Breeding in Crop Improvement
}

\author{
Temesgen Begna \\ Chiro National Sorghum Research and Training Center, P.O.BOX 190, Chiro, Ethiopia
}

\begin{abstract}
Molecular marker is applicable to many aspects of plant improvement and crop production. The main objective of plant breeding to produce crops with improved characteristics with the utilization of the available genetic variability and producing sufficient genetic variability of crops by different breeding techniques. There are possibilities to improve the desired traits through conventional breeding methods in the presence of genetic diversities. However, there are several challenges to make the significant improvement on the crop through conventional breeding. Conventional breeding is almost always based on phenotypic variation of the crops, which is affected by environments (non-heritable components) and crop improvement cycle takes long time. However, molecular marker is designed to meet this challenges regardless of its cost and it's not affected by environment where ever the experiment is conducted either in laboratory or field condition. Molecular marker procedures are playing a significant role to increase the effectiveness in breeding and shorten the development crop improvement stages. Molecular markers also used develop resistant crop to pests and diseases, develop tolerant crop to environmental conditions and improve the crop in required quality. In facing the challenge of improving several lines for quantitative traits, marker assisted selection strategies use DNA markers in one key selection step to maximize their impact. With the development of molecular marker technology, the fate of plant breeding has changed. Different types of molecular markers have been developed in identification and characterization germplasm, DNA sequences in identification of the genomic regions involved in the expression of the target traits, to analysis the genetic variation, cytogenetic, quantitative genetics, biotechnology and genomics and it's applied in genetic diversity analysis in crop improvement. It is possible to increase agricultural productivity through addressing the problems of yield reduction and its links with pest management and climate change using advanced breeding technologies. There are several major challenges in the application of molecular markers to agronomically important traits. Some of them are: economic factors, lack of grants to researchers, lack of adequately trained personnel. Generally, the integrating molecular marker technologies with the conventional breeding strategies are increasingly important to realize genetic gains with greater speed and precision.
\end{abstract}

Keywords: Molecular marker; Marker assisted breeding; quantitative traits; Plant breeding

DOI: $10.7176 / J N S R / 11-21-04$

Publication date: November $30^{\text {th }} 2020$

\section{Introduction}

Plant breeding is primarily depends on presence of substantial genetic variation to address the maximum genetic yield potential of the crops and exploitation of this variation through effective selection for improvement. The selection of plants from a population is almost always based on their phenotype and the phenotype has both heritable and non-heritable components. Plant breeding program plays a key role in increasing yield, disease and insect resistance, abiotic stress tolerance and to improve quality characteristics (Collard and Mackill, 2008). However, plant breeders are facing with major challenges in increasing crop production because of several constraints like global warming, creating new biotypes of diseases and insects and various abiotic stresses which significantly reduce crop yield (Ni, J., Colowit, P.M and Mackill DJ 2002). Additionally, there are different objections in crop improvement since the environment greatly affects the phenotypes and the genetic potential of the crop is also affected by environments.

The global food production must be increased by $50 \%$ to meet the projected demands of the world's population by 2050 since the world population is increasing in alert rate. Overcoming these difficult challenges will be harder in the absence international integration of plant biotechnology into plant improvement to increase agricultural productivity through addressing the problem of yield reduction and its links with pest management and climate change (Scholes and Biggs, 2004). The practice of improving crop production system with advanced breeding techniques play an important role to alleviate poverty and raise the living standards of the peoples by obtaining better yields of different crops (Gruhn et al., 2000).

The recent integration of advances in biotechnology, genomic research and molecular marker applications with conventional plant breeding practices has created the foundation for molecular plant breeding which is revolutionizing $21^{\text {st }}$ century crop improvement (Cooper et al., 2004). The typical crop improvement cycle takes 10-15 years to complete and includes germplasm manipulations, genotype selection and stabilization, variety testing, variety increase, proprietary protection and crop production stages. However, with the utilization of molecular marker procedures, it is possible to increase the effectiveness in breeding and to significantly shorten 
the development crop improvement stages. Molecular markers also used develop resistant crop to pests and diseases, develop tolerant crop to environmental conditions and improve the quality of crop. From its definition molecular plant breeding is the use of genetic manipulation performed at DNA molecular level to improve characters of interest in plants including genetic engineering, genomic selection and molecular marker assisted selection. Molecular marker also used to describe several modern breeding strategies, which includes markerassisted backcrossing, marker-assisted recurrent selection, and genome-wide selection (Ribaut et al., 2010).

Genetic marker is a biological marker that determined by allelic forms of genes and can be transmitted from one generation to another generation. Genetic markers primarily classified into two categories: these are classical markers and DNA markers (Xu, 2010). Classical markers include morphological markers, cytological markers and biochemical markers whereas DNA markers have developed into many systems based on different polymorphism detecting methods (DNA sequencing, southern blotting or nuclear acid hybridization, polymerase chain reaction, and DNA sequencing) (Collard et al., 2005). The progress made in biotechnology and genomics is preparing the path to meet the challenges; besides that new genes for resistance to major biotic and abiotic stresses are constantly being major biotic and abiotic identified using DNA markers.

Integration of desired genes from different backgrounds of elite cultivars led to the development of crops gene pool and the development of improved crop varieties suitable for different agro-climatic conditions. The objective/s of the paper was to review the challenges and progresses of molecular plant breeding in crop improvement and as well as to understand its role and speed in crop improvement strategies.

\section{Development of Molecular Plant Breeding}

Plant breeding has a long history of integrating the latest innovations in biology and genetics to enhance crop improvement. Prehistoric selection for visible phenotypes that facilitated increased productivity led to the domestication of the first crop varieties (Harlan, 1992). Traditional plant breeding involves cross-breeding of similar plants to produce new varieties with different traits. But it takes many generations to achieve desired result. By using various biotechnological tools, crop improvement can be achieved faster and it even facilitates to transfer genes from unrelated species (Paterson et al., 1988).

In recent years, novel breeding methods have been developed that allow the genetic material of crops to be adapted to particular growing conditions and to demand in a targeted manner that saves time (Delannay et al., 1995). Darwin outlined the scientific principles of hybridization and selection whereas, Mendel defined the fundamental association between genotype and phenotype, discoveries that enabled a scientific approach to plant breeding at the beginning of the $20^{\text {th }}$ century (Shull, 1910). By 1996, the commercialization of transgenic crops demonstrated the successful integration of biotechnology into plant breeding and crop improvement programs (Koziel et al., 1993; Delannay et al., 1995).

Recently, molecular marker is developed in order to understand to analysis the genetic variation, cytogenetic, quantitative genetics, biotechnology and genomics and it's applied in genetic diversity analysis in crop improvement (Baenziger et al., 2006; Jauhar, 2006; Varshney et al., 2006). Marker-assisted genetic analysis studies DNA sequences to identify genes, quantitative trait loci, and other molecular markers and to associate them with organism functions, i.e. gene identification. Marker-assisted selection is the identification and inheritance tracing of previously identified DNA fragments through a series of generations (Sharma et al., 2002; Varshney et al., 2006; Collard and Mackill, 2008). Marker assisted back crossing was developed for the introgression of transgenic traits, whereas molecular marker was used in genome scans to select those individuals that contained both the transgene and the greatest proportion of favorable alleles from the recurrent parent genome (Ragot et al., 1995).

\subsection{Plant Breeding for Crop Improvement}

Plant breeding is about the genetic improvement of crop through creation of genetic variability and selection of elite genotypes from that variability for desirable traits (John, 2002). Molecular technology contributed to the creation of genetic variation into two different ways: First, molecular-marker technology has provided a method of determining relationships among germplasm sources. Secondly, the potential gene pool for improving cultivated crops has been greatly broadened by the use of transgenic technology. Molecular markers have been used in a variety of crops to determine relationships among germplasm sources and played significant role in the characterization of germplasm, known as fingerprinting, identification of the genomic regions involved in the expression of the target traits and crop improvement through marker-assisted selection (Ribaut et al., 2002).

\subsection{Role of Quantitative Trait Loci in Plant Breeding Program}

There are many reasons to believe that plant breeding in the $21^{\text {st }}$ century will still depend on conventional methods for phenotypic selection. Molecular biology is used to improve recombinant frequency for favorable alleles and select the traits that are not measurable under normal environments with conventional methods. Many agriculturally important traits such as yield, quality and some forms of disease resistance are controlled by many 
genes and are known as quantitative traits (also polygenic, multifactorial or complex traits). The regions within genomes that contain genes associated with a particular quantitative trait are known as quantitative trait loci (QTLs) (Collard et al., 2005).

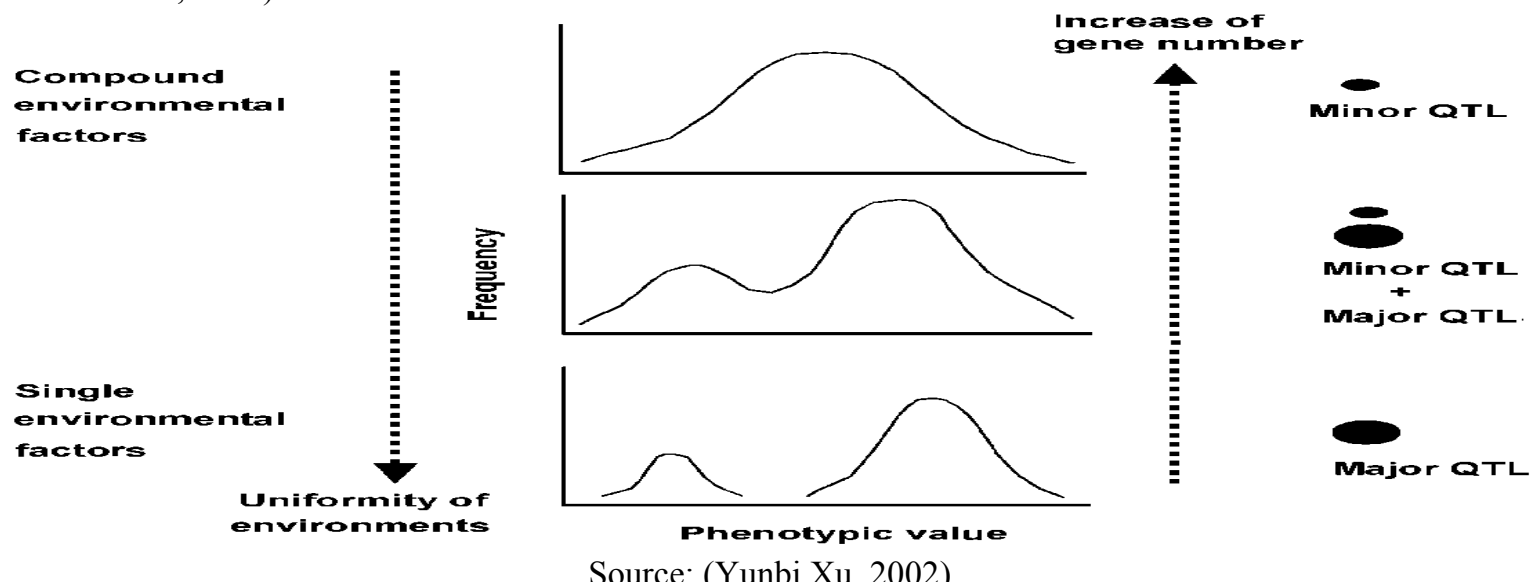

Figure 1: Relationship between phenotypes, genes and environments

\subsection{Principles and Practices of Molecular Plant Breeding}

\subsubsection{Breeding Schemes and Genetic Gain Concept}

An important concept in quantitative genetics and plant breeding is genetic gain, which is the predicted change in the mean value of a trait within a population that occurs with selection. Regardless of species, the trait of interest, or the breeding methods employed, genetic gain serves as a simple universal expression for expected genetic improvement (Falconer and Mackay, 1996).

\subsubsection{Role of Molecular Plant Breeding in Genetic Diversity}

The key prerequisite to successful plant breeding is the availability of genetic diversity. The most important issue in plant breeding is creating genetic diversity and manipulating genetic variability. Phenotypic variation is positively associated with genetic diversity, yet also depends on environmental factors and the interactions between genotype and environment (Buckler et al., 2006). A common first step in plant breeding is to create a genetically diverse population by crossing unrelated parents and by inducing mutations in the breeding germplasm (Smith and cooper, 2004). The difficulties that are associated with selecting plants that express recessive alleles, genes that have only minor effects on plant phenotypes or genes whose expression is strongly modified by the growth environment are major limiting factors in plant improvement. On this regard several biotechnological procedures have greatly expanded the possibilities for increasing the genetic diversity of crop species through molecular assisted selection of potential parents, tissue culture-induced variability, protoplast fusion and the production of transgenic plants (Smith and cooper, 2004). In a breeding program which uses the molecular markers, progeny from each mating cycle are selected on the basis of the presence of a particular electrophoretic band that has been shown to be tightly linked to the desired characteristic (Ralfalski \& Tingey, 1993).

Marker-assisted selection also allows early stage selection to be carried out since the genetic pattern does not change during plant development. Furthermore, after a linkage map of molecular markers has been constructed a whole genome selection procedure can be utilized, thus, decreasing the time required to fix a gene in an agronomically useful background (Paterson et al., 1988). Molecular makers have proven to be powerful tools in the assessment of genetic variation and in elucidation of genetic relationships within and among species (Chakravarthi and Naravaneni, 2006). Collecting DNA marker data to determine whether phenotypically similar cultivars are genetically similar would therefore be of great interest in crop breeding programme (Duzyaman, 2005). The use of molecular genetics for detecting differences in the DNA of individual plants has many applications of value to crop improvement (Chakravarthi and Naravaneni, 2006). 


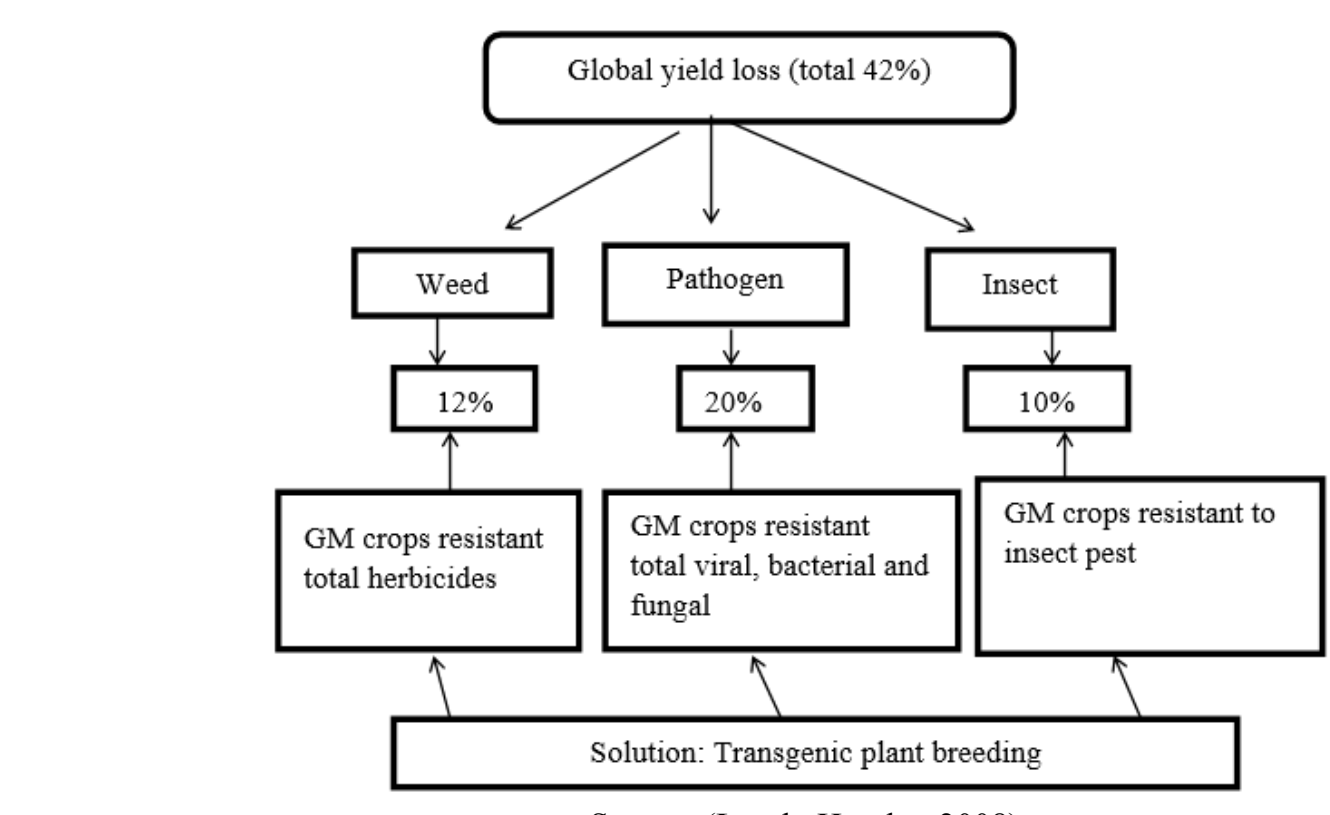

Source: (Laszlo Heszky, 2008)

Figure 2 : Global yield loss and the solution

\subsection{Genetic Markers}

In conventional plant breeding, genetic variation is usually identified by visual selection. However, with the development of molecular biology, it can now be identified at the molecular level based on changes in the DNA and their effect on the phenotype. Molecular changes can be identified by the many techniques that have been used to label and amplify DNA and to highlight the DNA variation among individuals. Once the DNA has been extracted from plants or their seeds, variation in samples can be identified using a polymerase chain reaction (PCR) and/or hybridization process followed by polyacrylamide gel electrophoresis (PAGE) or capillary electrophoresis (CE) to identify distinct molecules based on their sizes, chemical compositions and charges. Genetic markers are used to tag and track genetic variation in DNA samples. Genetic markers are biological features that are determined by allelic forms and can be used as experimental probes or tags to keep track of an individual, a tissue, cell, nucleus, chromosome or gene $(\mathrm{Xu}, 2010)$. In classical genetics, genetic polymorphism represents allelic variation.

In modern genetics, genetic polymorphism is the relative difference at any genetic locus across a genome. Desirable genetic markers should meet the following criteria: (i) high level of genetic polymorphism; (ii) codominance (so that heterozygote can be distinguished from homozygote); (iii) clear distinct allele features (so that different alleles can be identified easily); (iv) even distribution on the entire genome; (v) neutral selection (without pleiotropic effect); (vi) easy detection (so that the whole process can be automated); (vii) low cost of marker development and genotyping; and (viii) high duplicability (Xu ,2010). Genetic markers used in genetics and plant breeding can be classified into two categories: classical markers and DNA markers (Xu, 2010). Classical markers include morphological markers, cytological markers and biochemical markers. DNA markers have developed into many systems based on different polymorphism-detecting techniques or methods like polymerase chain reaction, and DNA sequencing) (Firas Rashad, 2015), like, RFLP, AFLP, RAPD, SSR, SNP.

\subsubsection{Classical Markers}

Morphological Markers

Any phenotypic difference controlled by genes, that can be used for studying recombination process or selection of a more or less closely associated target gene. Mendel has proposed two basic rules of genetics which were the laws of segregation and independent assortment. Mendel selected individuals which differed in a particular trait and used them as the parental lines in a cross breeding experiment to determine the phenotype of the offspring with regard to the selected trait. The plants in the segregated populations of the pea, such as $\mathrm{F}_{2}$ and backcross, were classified into two distinct groups depending on their phenotypes. These contrasting morphological phenotypes are the starting point for any genetic analysis and can be mapped to particular chromosomes using the Mendelian laws of inheritance and can thus be used as morphological markers of the genome and the particular trait. Morphological markers therefore generally represent genetic polymorphisms which are visible as differences in appearance, such as the relative difference in plant height and color, distinct differences in response to abiotic and biotic stresses, and the presence/absence of other specific morphological characteristics (Khush, 1987). 
Cytological Markers

By studying the morphology, number and structure of chromosomes from different species, particular cytogenetic characteristics can be found, such as various types of aneuploidy, variants of chromosome structure and abnormal chromosomes. These can be used as genetic markers to locate other genes on to chromosomes and determine their relative positions, or used for genetic mapping via chromosome manipulations such as chromosome substitution. The structural features of chromosomes can be shown by chromosome karyotype and bands. The banding patterns are indicated by color, width, order and position, revealing the difference in distributions of euchromatin and heterochromatin. Cytological markers have been widely used to identify linkage groups within specific chromosomes and have been widely applied in physical mapping. However, because of the limited number and resolution, they have limited applications in genetic diversity analysis, genetic mapping and marker-assisted selection (XU, 2010).

Protein Markers

Isozymes can be genetically mapped on to chromosomes and then used as genetic markers for mapping other genes. This can be used to measure population subdivision, genetic diversity, gene flow, genetic structure of species, and comparisons among species out-crossing rates, population structure and population divergence. Isozyme markers are based on their biochemistry and thus are also known as biochemical or protein markers. However, their use as markers is limited. Each isozyme can only be identified with a specific stain which also limits their use in practice (Vallegos and Chase, 1991).

2.4.2 DNA Markers

Molecular markers is very useful in crop genetic studies of assessment of genetic variability and characterization of germplasm, identification and fingerprinting of genotypes, estimation of genetic distances between population, inbreeds, and breeding materials, detection of monogenic and quantitative trait loci, identification of sequences of useful candidate genes. Basic DNA marker techniques can be classified into two categories. These are (1) PCRbased techniques and (2) non-PCR-based techniques or hybridization based techniques.

Restriction Fragment Length Polymorphisms (RFLP) is the first and forest molecular markers system called the restriction fragment length polymorphism. Restriction fragment length polymorphism is non-polymerase chain reaction based genetic markers and it was developed in early 1980 (Farooq and Azam, 2002). Genetic information is stored in the DNA sequence on a chromosome and variation in this sequence is the basis for the genetic diversity within species. Plants are able to replicate their DNA with high accuracy and rapidity, but many mechanisms causing changes (mutation) in the DNA operative (Joshi et al., 2011). This leads to simple or large-base pair changes as a result of inversion, translocation, transpositions or deletion which may occur, resulting in a loss or gain of a recognition sites and in turn lead to restriction fragment of different lengths. In this method, DNA is digested with restriction enzyme like EcoR1, which cut the DNA at specific sequences, electrophoresed, blotted on a membrane and probed with a labeled clone. Gel electrophoresis: digested products (restriction fragments) are electrophoresed on agarose gel and when visualized appear as smears because of the large number of fragments. The agarose gel is denatured using $\mathrm{NaOH}$ solution and then neutralized. The DNA fragments are transferred to a nitrocellulose membrane using Southern blotting.

Amplified Fragment Length Polymorphism (AFLP) is a PCR based marker with 10-12 base pair primers and amplified fragment length polymorphisms are fragments of DNA that have been amplified using directed primers from restriction of genomic DNA (William et al., 1991). In this approach the sample DNA is enzymatically cut up into small fragments (as with restriction fragment length polymorphism analysis), but only a fraction of fragments are studied following selective polymerase chain reaction amplification (Liu et al., 1994). Amplified fragment length polymorphism technique shares some characteristic with both restriction fragment length polymorphism and randomly-amplified polymorphic DNA analysis (Farooq and Azam, 2002) and combines the specifically of restriction analyses with polymerase chain reaction amplification. The major advantages of amplified fragment length polymorphism techniques are: generation of a large number of polymorphism. No sequence information is required. The polymerase chain reaction technique is fast with high multiplex ratio which makes the amplified fragment length polymorphisms very attractive choice (Farooq and Azam, 2002). Randomly amplified polymorphic DNA marker is the randomly amplified polymorphic DNA marker (RAPD) detects nucleotide sequence polymorphism in DNA by using a single primer of arbitrary nucleotide sequence (William et al., 1991).

In this reaction, a single species of primer anneals to the genomic DNA at two different sites on complementary strands of DNA template. Advantages associated with randomly-amplified polymorphic DNA analysis include: (i) Use of small amount of DNA which makes it possible to work with population that is not accessible with restriction fragment length polymorphism. It is fast and efficient in analysis having high-density genetic mapping as in many plant species (Kiss et al., 1993), (ii) Non-involvement with radioactive assays (Kiss et al., 1993) (iii) Non-requirement of species specific probe librararies (IV) Non-involvement in blotting or hybridization. Limitations of randomly-amplified polymorphic DNA markers are: (i) its polymorphisms are inherited as dominant or recessive characters causing a loss of information relative to markers which show co- 
dominance. (ii) Primers are relatively short, a mismatch of even a single nucleotide can often prevent the primer from annealing, hence leads to a loss of band. (iii) Suffers from problems of repeatability in many systems, especially when transferring between populations or laboratories as is frequently necessary with marker assisted selection programs (Liu et al., 1994).

Simple Sequence Repeats (SSR) referred to as simple sequence length polymorphisms and it's abundant and is relatively evenly spaced throughout eukaryotic genomes (Tautz and Renz, 1984). Simple sequence repeats loci are extremely variable in the number of repeat units among individuals of a given species. One of the most important attributes of microsatellite loci is their high level of allelic variation, making them valuable as genetic markers. The unique sequences bordering the simple sequence repeat motifs provide templates for specific primers to amplify the simple sequence repeat alleles via polymerase chain reaction (Freudenreich et al., 1997). These are ideal genetic markers for detecting differences between and within species of genes of all eukaryotes (Farooq and Azam, 2002).

It consist of tandemly repeated 2-7 base pair units arranged in repeats of mono-, di-, tri-, tetra and pentanucleotides (A,T, AT, GA, AGG, AAAG etc) with different lengths of repeat motifs. These repeats are widely distributed throughout the plants and animal genomes that display high level of genetic variation based on differences in the number of tandemly repeating units of a locus. The variation in the number of tandemly repeated units results in highly polymorphic banding pattern (Farooq and Azam, 2002) which are detected by polymerase chain reaction, using locus specific flanking region primers where they are known. Some of the prominent features of these markers are that they are dominant fingerprinting markers and Codominant sequence tagged microsatellites (STMS) markers (Joshi et al., 2011).

Single nucleotide polymorphism (SNP) is a single nucleotide polymorphism or SNP (pronounced snip) is an individual nucleotide base difference between two DNA sequences. Single nucleotide polymorphism can be categorized according to nucleotide substitution as either transitions $(\mathrm{C} / \mathrm{T}$ or $\mathrm{G} / \mathrm{A})$ or transversions $(\mathrm{C} / \mathrm{G}, \mathrm{A} / \mathrm{T}, \mathrm{C} / \mathrm{A}$ or $\mathrm{T} / \mathrm{G}$ ). In practice, single base variants in cDNA (mRNA) are considered to be Single nucleotide polymorphisms as are single base insertions and deletions (indels) in the genome. As a nucleotide base is the smallest unit of inheritance, Single nucleotide polymorphisms provide the ultimate form of molecular marker. For a variation to be considered a Single nucleotide polymorphism, it must occur in at least 1\% of the population. Based on partial genomic sequence information, Single nucleotide polymorphism frequencies have been revealed in many crops, including barley, soybean, sugarbeet, maize, cassava and potato; typical Single nucleotide polymorphism frequencies are also in the range of one Single nucleotide polymorphism every 100 - $300 \mathrm{bp}$ in plants (Edwards et $a l .$, 2007). Single nucleotide polymorphisms may fall within coding sequences of genes, non-coding regions of genes or in the intergenic regions between genes at different frequencies in different chromosome regions (Schmid et al., 2003).

TABLE 1: COMPARISON OF MOST COMMONLY USED MARKER SYSTEMS (ADOPTED FROM KORZUN, 2003)

\begin{tabular}{|l|l|l|l|l|l|l|}
\hline Marker & Reproducibility & $\begin{array}{l}\text { Technical } \\
\text { difficulty }\end{array}$ & Inheritance & $\begin{array}{l}\text { Sequence } \\
\text { Information }\end{array}$ & $\begin{array}{l}\text { PCR- } \\
\text { based }\end{array}$ & $\begin{array}{l}\text { DNA } \\
\text { quality }\end{array}$ \\
\hline RFLP & High & High & Codominant & Not required & No & High \\
\hline SSR & High & Medium & Codominant & Required & yes & High \\
\hline RAPD & Low & Low & Dominant & Not required & yes & Moderate \\
\hline AFLP & High & Medium & Codominant & Not required & yes & Moderate \\
\hline SNP & High & High & Codominant & Required & yes & High \\
\hline
\end{tabular}

Source: Current Technologies in Plant Molecular Breeding, 2015

\subsection{Advantages of Marker Assisted Breeding}

Classical plant breeding is the intentional interbreeding and selection of plant varieties with the goal of producing new varieties with improved properties (e.g. higher yield, bigger seed size, disease resistance, etc.). The use of DNA markers for screening and selecting of plants in a breeding program provides several advantages and is therefore very attractive to plant breeders. As DNA marker based genotypes can be obtained from almost any plant tissue, plants can be screened already at the seedling stage or even as seeds, thus allowing early selection for traits which may be expressed in matured plants only (i.e. grain or fruit quality, male sterility, photoperiod sensitivity). With the availability of preflowering genotypic information marker assisted selection allows for controlled pollination. Target alleles that are difficult, expensive and time consuming to score phenotypically can be selected with the assistance of markers (e.g. environmentally sensitive traits, as DNA markers are mostly neutral to environmental variation). In backcrossing, DNA markers can help to minimize linkage drag around the target gene and reduce the generations required to recover a recurrent parent's genetic background. In the choice of parents in crossing programs markers can be applied too. Here they can either help to maximize diversity, and in this way support the exploitation of heterosis (Maria R. Finckh, 2010). 


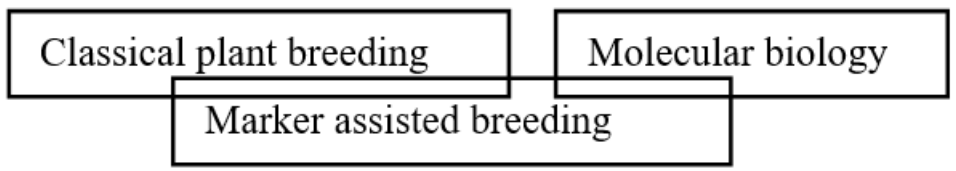

\subsection{Application of molecular marker in plant breeding program}

\subsubsection{Marker Assisted Back Crossing}

Backcrossing is used in plant breeding to transfer (introgress) favorable traits from a donor plant into an elite genotype (recurrent parent). In repeated crossings the original cross is backcrossed with the recurrent parent until most of the genes stemming from the donor are eliminated (Becker, 1993). However, the donor segments attached to the target allele can remain relatively large, even after many backcrossing generations. In order to minimize this linkage drag, marker assays can be of advantage (Frisch et al., 1999). Markers can be used in the context of marker assisted back crossing to either control the target gene or to accelerate the reconstruction of the recurrent parent genotype. According to Tanksley et al., (1989), in traditional backcross breeding the reconstruction of the recurrent parent genotype requires more than six generations, while this may be reduced to only three generations in marker assisted back crossing.

These findings are confirmed by results of Frisch et al. (1999), who showed in a computer simulation that marker assisted selection can reconstruct a level of recurrent parent genome in back cross three which would only be reached in back cross seven without the use of markers. However, it has to be taken into account that backcrossing is a very conservative breeding strategy and should not become the prime focus of a breeding program (Eathington et al., 2007). At the moment marker assisted back crossing also is and will probably remain the preferred means of backcrossing transgenes into elite inbred lines, which is also considerably contributing to its popularity (Ragot \& Lee, 2007).

\subsubsection{Marker Assisted Recurrent Selection}

Marker assisted recurrent selection include identification and selection of several genomic regions (up to 20 or even more) for complex traits within a single population. The improvement of complex traits via phenotypic recurrent selection is generally possible, but the long selection cycles impose restrictions on the practicability of this breeding method. With the use of markers, recurrent selection can be accelerated considerably. Several selection-cycles are possible within one year, accumulating favorable quantitative trait loci alleles in the breeding population (Eathington et al., 2007).

\subsection{Molecular Maps}

The order and relative distance of genetic features that are associated with genetic variation or polymorphisms can be determined by genetic mapping. Genetic maps constructed using molecular markers can also be used to locate major genes which can then also be used as genetic markers.

\subsection{Molecular Plant Breeding Challenges and Perspectives}

Marker-assisted breeding became a new member in the family of plant breeding as various types of molecular markers in crop plants were developed during the 1980s and 1990s. The extensive use of molecular markers in various fields of plant science, e.g. germplasm evaluation, genetic mapping, map-based gene discovery, characterization of traits and crop improvement, has proven that molecular technology is a powerful and reliable tool in genetic manipulation of agronomically important traits in crop plants. Compared with conventional breeding methods, marker assisted back cross has significant advantages: marker assisted back cross can allow selection for all kinds of traits to be carried out at seedling stage and thus reduce the time required before the phenotype of an individual plant is known. For the traits that are expressed at later developmental stages, undesirable genotypes can be quickly eliminated by marker assisted selection. This feature is particularly important and useful for some breeding schemes such as backcrossing and recurrent selection, in which crossing with or between selected individuals is required.

Marker assisted back cross can be not affected by environment, thus allowing the selection to be performed under any environmental conditions (e.g. greenhouse and off-season nurseries). This is very helpful for improvement of some traits (e.g. disease or pest resistance and stress tolerance) that are expressed only when favorable environmental conditions present. For low-heritability traits that are easily affected by environments, marker assisted selection based on reliable markers tightly linked to the multiple traits for traits of interest can be more effective and produce greater progress than phenotypic selection. Marker assisted back cross using codominance markers can allow effective selection of recessive alleles of desired traits in the heterozygous status. No selfing or test crossing is needed to detect the traits controlled by recessive alleles, thus saving time and accelerating breeding progress. For the traits controlled by multiple genes individual genes can be identified and selected in marker assisted back cross at the same time and in the same individuals, and thus marker assisted back 
cross is particularly suitable for gene pyramiding. In traditional phenotypic selection, however, to distinguish individual genes or loci is problematic as one gene may mask the effect of additional genes. Genotypic assays based on molecular markers may be faster, cheaper and more accurate than conventional phenotypic assays, depending on the traits and conditions, and thus marker assisted back cross may result in higher effectiveness and higher efficiency in terms of time, resources and efforts saved (Collard and Mackill, 2008; Wang and Chee, 2010). The application of molecular markers in plant breeding has not achieved the results as expected previously in terms of extent and success (Collard and Mackill, 2008). The application of molecular technologies to plant breeding is still facing the following drawbacks and/or challenges: Not all markers are breeder-friendly. This problem may be solved by converting of nonbreeder- friendly markers to other types of breeder-friendly markers (e.g. RFLP to STS, sequence tagged site, and RAPD to SCAR, sequence characterized amplified region). Not all markers can be applicable across populations due to lack of marker polymorphism or reliable marker-trait association. Multiple mapping populations are helpful in understanding marker allelic diversity and genetic background effects.

In addition, quantitative trait loci positions and effects also need to be validated and re-estimated by breeders in their specific germplasm (Heffner et al., 2009). False selection may occur due to recombination between the markers and the genes of interest. Use of flanking markers or more markers for the target gene or quantitative trait loci can help. Imprecise estimates of quantitative trait loci locations and effects result in slower progress than expected. The efficiency of quantitative trait loci detection is attributed to multiple factors, such as algorithms, mapping methods, number of polymorphic markers, and population type and size (Wang et al., 2012). High marker density fine mapping with large populations and well-designed phenotyping across multiple environments may provide more accurate estimates of quantitative trait loci location and effects. A large number of breeding programs have not been equipped with adequate facilities and conditions for a large-scale adoption of marker assisted backcross in practice. The methods and schemes of marker assisted back crossing must be easily understandable, acceptable and implementable for plant breeders, unless they are not designed for a large scale use in practical breeding programs. Higher start-up expenses and labor costs.

\subsection{Challenges to the Application of Molecular Markers}

Economic factors: Economics is the key determinant for the application of molecular genetics in genetic improvement programmes (Ebegbulem, 2013). The use of markers in selection incurs the costs that are inherent to molecular techniques. Developing costs (e.g. identifying molecular markers on the genome, detecting association between markers and the traits of interest) and running costs. Besides, the cost of importation of the technology from developed countries could be so outrageous that it may out-weigh whatever benefits that could be derived from it. Mechanical and Logistics factors: In Africa presently, functional biotechnological and genomic Centers are not very common. Molecular markers have to be imported from countries like the USA and the UK, researchers have to place orders long in advance when the need to use such markers arise ,and the delivery of these markers to their point of use may take several days. This long delays impacts negatively on the potency of the imported markers, which consequently complicate or distort experimental results.

Lack of funds or grants to researchers: The researches involving molecular technologies are being hampered in developing nations due to the inability of researchers to access grants and funds. Researchers are denied opportunity to secure research grants because their institutions or their basic affiliations could not provide the basic equipment or facilities required to effectively carry out. Sometimes when research grants are provided, the amount is hardly sufficient to procure all the necessary reagents and other consumables. But, it is common knowledge that meaningful research especially molecular studies require a lot of funds. Erratic power supply: Erratic power supply appears to be the most challenging factor impeding human activities in developing countries. Molecular markers need very cool environment at all times and storage materials like refrigerators and deep freezers connected to a regular supply of electricity is necessary, as markers devoid of a cooler environment will not work when employed in polymerase chain reaction technology. Lack of adequately trained personnel: The application of molecular markers to the improvement of animal species in developing country is also being hampered by the nonavailability of enough number of adequately trained personnel with the requisite practical experience in the Universities. Some who are well trained have been rendered redundant because of non-motivation, while others have opted to move to the developed countries to work.

\section{Conclusion}

The fundamental discoveries of Darwin and Mendel established the scientific basis for plant breeding and genetics at the turn of the $20^{\text {th }}$ century. The general goal of plant breeding is the improvement of plants for human benefit, fulfilling the needs of both producers and consumers. However, the exponential growth of human population, the effects of the global warming on the crop environments, several biotic and abiotic stresses and the need to stop the negative impact of agriculture on the ecosystems, are demanding new and urgent specific goals to maintain the quality of life on earth. To meet growing need of ever increasing human population, we need to enhance food production for sustaining food supply. In the genomics era, advances in molecular biology have opened new 
opportunities to accelerate plant breeding processes and in overcoming the above constraints limiting crop productivity. Molecular markers have become important tools in the hands of plant breeders in marker assisted breeding and for enhancing the selection efficiency for various agronomic traits in precision plant breeding. The isolation, cloning and moving of genes from diverse biological sources into plant genomes holds promise to broaden the gene pool of crops and develop new plant varieties for specific traits that determine yield, quality, and resistance to biotic and abiotic stresses. New genomics tools will be of great value to support conventional breeding for sustainable food production especially under the climate change and meet demand of ever growing human population.

A noteworthy research in conventional breeding for several years has made the world self-sufficient in many respects. However, the ever increasing population has alarmed food security in the world and attempts have been initiated to integrate modern biotechnology tools in conventional breeding to improve the most important crops. Regardless of its wider utilization and application worldwide, science and technology is playing a very important role in overcoming the potential problems of agriculture. The integration of conventional plant breeding with various biotechnological techniques advance crop genetic improvement and shortening the crop improvement cycle with desirable traits in order to satisfy the demand of people in both quantitative and qualitative. Molecular breeding implies the application of molecular biotechnologies, specifically molecular markers in combination with linkage maps and genomics are playing the key role to improve plant traits on the basis of genotypic assays. Genetic markers are the biological features that are determined by allelic forms of genes or genetic loci which can be transmitted from one generation to another.

Generally, the world population is increasing alarmingly, but productivity is reduced because of several production challenges. Hence, conventional plant breeding method alone cannot address the serious challenges that world facing. Therefore, in order to overcome the food security problems, conventional plant breeding should be assisted and integrated with various biotechnology developments to hasten the crop genetic improvements. To ensure the rapid and advanced agricultural developmental within short period of time, the incorporation of molecular marker in plant breeding very relevant in the future world. Marker assisted breeding is not about the replacement of conventional breeding rather than integrating with it in order to make further improvement. There are various challenges in relation to the application of molecular markers because of its costs in large scale utilization especially in developing countries. The integration of marker assisted breeding into conventional breeding program is an optimistic strategy for crop improvement in the future.

\section{REFERENCES}

[1] B.C, Collard, and D.J, Mackill, Marker-assisted selection: an approach for precision plant breeding in the twenty-first century. Philosophical Transactions of the Royal Society B: Biological Sciences, 2008, pp.557572 .

[2] J, Ni., P.M, Colowit, and D.J, Mackill, Evaluation of genetic diversity in rice subspecies using microsatellite markers. Crop science, 2002, pp.601-607.

[3] R, Biggs., R.J, Scholes, and B, Reyers, Assessing biodiversity intactness multiple scales. Bridging scales and epistemologies: linking local knowledge and global science in multi-scale assessment. March. Alexandria, Egypt, 2004.

[4] P, Gruhn., R.L, Paarlberg,., F, Goletti, F. and M, Yudelman, Governing the GM crop revolution: policy choices for developing countries, 2000.

[5] M, Cooper., O.S, Smith.., G, Graham, and A, Lane, A., 2004. Genomics, genetics, and plant breeding: a private sector perspective. Crop Science, 44(6), p.1907.

[6] J.M, Ribaut., M.C, De Vicente, and X, Delannay, Molecular breeding in developing countries: challenges and perspectives. Current opinion in plant biology, 2010, pp.213-218.

[7] Y, Xu, Molecular plant breeding. Cabi, 2010.

[8] B.C, Collard, M.Z, Jahufer., J.B, Brouwer, and E.C.K, Pang, An introduction to markers, quantitative trait loci (QTL) mapping and marker-assisted selection for crop improvement: the basic concepts. Euphytica, 2005, pp.169-196.

[9]J.R, Harlan, Origins and processes of domestication. Grass evolution and domestication, 1992, pp.175.

[10] C, Patterson, Homology in classical and molecular biology. Molecular biology and evolution, 1988, pp.603625.

[11] D.E, Delaney., S, Nasuda., T.R, Endo., B.S, Gill, and S.H, Hulbert, Cytological based physical maps of the group-2 chromosomes of wheat. Theoretical and Applied Genetics, 1995, pp.568-573.

[12] G.H, Shull, Hybridization methods in corn breeding. Journal of Heredity, 1910, pp.98-107.

[13] M.J, Koziel.,G.L, Beland.,C, Bowman., N.B, Carozzi., R, Crenshaw., L, Crossland,., J, Dawson.,N, Desai.,M, Hill., S, Kadwell, and K, Launis,. Field performance of elite transgenic maize plants expressing an insecticidal protein derived from Bacillus thuringiensis. Bio/technology, 1993, pp.194-200.

[14] P.P, Jauhar, Cytogenetic architecture of cereal crops and their manipulation to fit human needs: Opportunities 
and challenges. In Genetic resources, chromosome engineering, and crop improvement, 2006, pp. 17-48.

[15] P.S, Baenziger.,W.K, Russell.,G.L,Graef, and B.T, Campbell, Improving lives. Crop science, 2006, pp.22302244.

[16] R.K, Varshney., D.A, Hoisington, and A.K, Tyagi, Advances in cereal genomics and applications in crop breeding. Trends in biotechnology, 2006, pp.490-499.

[17] A.D, Sharma., P.K, Gill, and P, Singh, DNA isolation from dry and fresh samples of polysaccharide-rich plants. Plant Molecular Biology Reporter, 2002, pp.415-415.

[18] M, Ragot., P.H, Sisco., D.A, Hoisington, and C.W, Stuber, Molecular - marker - mediated characterization of favorable exotic alleles at quantitative trait loci in maize. Crop science, 1995, pp.1306-1315.

[19] E.S, John.,N.L, Mahoney.,M.D, Hayward., I.P, Armstead., J.G, Jones.,M.O, Humphreys., I.P, King., T, Kishida., T, Yamada., F, Balfourier, and G, Charmet, An enhanced molecular marker based genetic map of perennial ryegrass (Lolium perenne) reveals comparative relationships with other Poaceae genomes. Genome, 2002, pp.282-295.

[20] J.M, Ribaut., M, Bänziger.,J, Betran.,C, Jiang, and G.O, Edmeades, Breeding: Drought Tolerance Improvement in Tropical Maize. Quantitative genetics, genomics, and plant breeding, 2002, pp.85.

[21] Y, Xu Global view of QTL: rice as a model. Quantitative genetics, genomics and plant breeding. CABI Publishing, Wallingford, 2002, pp.109-134.

[22] D. S, Falconer, and T.F, Mackay,. Introduction to quantitative genetics. Genetics, 2004, pp.1529-1536.

[23] E.S, Buckler., B.S, Gaut, and M.D, McMullen, Molecular and functional diversity of maize. Current opinion in plant biology, 2006, pp.172-176.

[24] D.N, Smith, and M, Cooper, Changes in performance, parentage, and genetic diversity of successful corn hybrids, 1930-2000. Corn Orig Hist Technol Prod, 2004, pp.65.

[25] A, Paterson., S.D, Tanksley., J, Miller, and R, Bernatzky, Molecular mapping of plant chromosomes. In Chromosome structure and function, 1988, pp. 157-173.

[26] A, Rafalski, and S, TingeY,. Genetic mapping and variability of seven soybean simple sequence repeat loci. Genome, 1994, pp.763-769.

[27] B.K, Chakravarthi, and R, Naravaneni,. SSR marker based DNA fingerprinting and diversity study in rice (Oryza sativa. L). African Journal of Biotechnology, 2006,PP. 9.

[28] E, Düzyaman. Phenotypic diversity within a collection of distinct okra (Abelmoschus esculentus) cultivars derived from Turkish land races. Genetic Resources and Crop Evolution, 2005, pp.1019-1030.

[29] L, Heszky, Challenges of plant breeding early in 21th century. Hungarian Agric Res, 2008, pp.4-8.

[30] J, Xu.,Q, Zhao.,P, Du.,C, Xu., B, Wang.,Q, Feng.,Q, Liu., S, Tang., M, Gu.,B, Han, and G, Liang, Developing high throughput genotyped chromosome segment substitution lines based on population whole-genome resequencing in rice (Oryza sativa L.). BMC genomics, 2010, p.656.

[31] G.S, Khush, Association of quantitative trait loci for plant height with major dwarfing genes in rice. Heredity, 1987, pp.130-137.

[32] C.E,Vallejos.,C.D, Chase, and V.M, Ortega, DNA restriction fragment length polymorphisms correlate with isozyme diversity in Phaseolus vulgaris L. Theoretical and applied genetics, 1991, pp.806-811.

[33] S, Farooq, and F, Azam, Molecular markers in plant breeding-II. Some pre-requisites for use. Pakistan journal of biological Sciences, 2002, pp.1141-1147.

[34] S.G, Joshi.,J.G, Schaart., R, Groenwold., E, Jacobsen., H.J, Schouten, and F.A, Krens, Functional analysis and expression profiling of HcrVf1 and HcrVf2 for development of scab resistant cisgenic and intragenic apples. Plant molecular biology, 2011, pp.579-591.

[35] Z, Liu., P, Li.,H, Kucuktas.,A, Nichols.,G, Tan., X, Zheng.,B,J, Argue., R.A, Dunham, and D.R, Yant,. Development of amplified fragment length polymorphism (AFLP) markers suitable for genetic linkage mapping of catfish. Transactions of the American Fisheries Society, 1994, pp.317-327.

[36] M.N.V, Williams., N, Pande., S, Nair., M, Mohan, and J, Bennett, Restriction fragment length polymorphism analysis of polymerase chain reaction products amplified from mapped loci of rice (Oryza sativa L.) genomic DNA. Theoretical and Applied Genetics, 1991, pp.489-498.

[37] G.B, Kiss., G, Csanadi,K, Kálmán., P, Kaló, and L, Okrész, Construction of a basic genetic map for alfalfa using RFLP, RAPD, isozyme and morphological markers. Molecular and General Genetics MGG, 1993, pp.129-137.

[38] D, Tautz, and M, Renz, Simple sequences are ubiquitous repetitive components of eukaryotic genomes. Nucleic acids research, 1984, pp.4127-4138.

[39] C.H, Freudenreich., J.B, Stavenhagen, and V.A, Zakian, Stability of a CTG/CAG trinucleotide repeat in yeast is dependent on its orientation in the genome. Molecular and cellular biology, 1997, pp.2090-2098.

[40] D, Edwards., J.W, Forster., D, Chagné, and J, Batley. What Are SNPs. In Association mapping in plants, 2007, pp. 41-52.

[41] K.J, Schmid., T.R, Sörensen., R, Stracke.,O, Torjek., T, Altmann.,T, Mitchell-Olds, and B, Weisshaar , Large- 
scale identification and analysis of genome-wide single-nucleotide polymorphisms for mapping in Arabidopsis thaliana. Genome research, 2003, pp.1250-1257.

[42] R, Maria, Finckh, Applications and potentials of marker assisted selection (MAS) in plant breeding. Final report of the F+ E project "Applications and Potentials of Smart Breeding, 2010.

[43] H.C, Becker.,H, Loptien, and G, Robbelen, Breeding: An overview. In Developments in plant genetics and Breeding, 1993, pp. 413-460).

[44] S.D, Tanksley., N, D, Young., A.H, Paterson, and M.W, Bonierbale, RFLP mapping in plant breeding: new tools for an old science. Bio/technology, 1989, pp.257-264.

[45] M, Frisch., M, Bohn, and A.. E, Melchinger, Comparison of selection strategies for marker-assisted backcrossing of a gene. Crop Science, 1999, pp.1295-1301.

[46] M, Ragot., M, Lee, and E, Guimaraes, Marker-assisted selection in maize: current status, potential, limitations and perspectives from the private and public sectors. Marker-assisted selection, Current status and future perspectives in crops, livestock, forestry and fish, 2007, pp.117-150.

[47] S.R, Eathington.,T.M, Crosbie., M.D, Edwards., R.S, Reiter, and J.K, Bull, Molecular markers in a commercial breeding program. Crop Science, 47(Supplement, 2007, pp.S-154.

[48] V.N, Ebegbulem, and P.O, Ozung, Application of molecular markers in farm animal improvement: Prospects and challenges. Online J Anim Feed Res, 2013, pp.149-152.

[49] E.L, Heffner., M.E, Sorrells, and J.L.,Jannink, Genomic selection for crop improvement. Crop Science, 2009, pp.1-12.

[50] H.M, Wang., W, Chen., X.R, Kong.,Y.L, Zhao.,Y.H, Li.,H.Y, Gong, and X.H, Sang.,. Effect of molecular marker assisted selection to Verticillium wilt resistance in upland cotton breeding. Cotton Sci, 2010, pp.527532.

[51] W, Wang., S, Huang., Y, Liu., Z, Fang., L, Yang., W, Hua., S, Yuan., S, Liu., J, Sun., M, Zhuang, and Y, Zhang, Construction and analysis of a high-density genetic linkage map in cabbage (Brassica oleracea L. var. capitata). BMC genomics, 2012, pp.523.

\section{AUTHOR'S PROFILE}

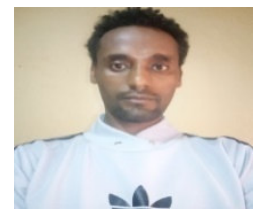

Temesgen Begna Chimdessa

MSc. In Plant Breeding 